\title{
Comparative study between four ultrasound Shear Waves Elastographic methods for liver fibrosis assessment
}

\author{
Ioan Sporea, Ruxandra Mare, Raluca Lupușoru, Alina Popescu, Mirela Danilă, Felix Bende, \\ Roxana Șirli
}

Department of Gastroenterology and Hepatology, "Victor Babeș” University of Medicine and Pharmacy, Timișoara, Romania

\begin{abstract}
Aims: Non-invasive methods to assess the severity of chronic liver diseases have become more and more popular. The aim of our study was to evaluate the correlation of four Shear Waves Elastographic methods in a cohort of patients with chronic liver diseases of mixed etiologies and to compare their performances for liver fibrosis evaluation. Material and methods: A prospective study was conducted on 127 consecutive patients with chronic liver diseases of mixed etiologies. LS measurements were performed in all subjects, in one session, during the same day, by means of 4 elastographic methods: Transient Elastography (TE), Virtual Touch Quantification (VTQ), ElastPQ and 2D-SWE.SSI. The diagnosis accuracies of VTQ, ElastPQ, 2D-SWE.SSI were then compared using TE as reference method. Results: Valid LS measurements by all four shear waves ultrasound elastographic methods were obtained in 116/127 subjects with VTQ, in 108/127 subjects with 2D-SWE.SSI, in 111/127 with TE and in 109/127 with ElastPQ, so the final analysis included 82/127 subjects (64.5\%). A good and significant correlation was found between all 4 elastographic methods. The diagnostic accuracy of VTQ, 2D-SWE.SSI and ElastPQ for the diagnosis of significant and severe fibrosis (F2/F3), and liver cirrhosis were similar for all elastographic techniques: VTQ vs 2D-SWE.SSI vs ElastPQ: $84.1 \%$ vs $85.3 \%$ vs. $84 \%$ ( $>>0.05$ ); $93.9 \%$ vs $94 \%$ vs $94 \%$ ( $>>0.05$ ). Conclusion: considering TE as the reference method for liver fibrosis evaluation, VTQ, ElastPQ, and 2D-SWE.SSI obtained similar accuracies for diagnosing at least significant fibrosis $(\mathrm{F} \geq 2)$ and liver cirrhosis.
\end{abstract}

Keywords: liver fibrosis; SWE; liver stiffness; cirrhosis

\section{Introduction}

Chronic liver diseases with different etiologies [viral chronic hepatitis (B or C), excessive alcohol consumption (alcoholic steatohepatitis), non alcoholic steatohepatitis (NASH), autoimmune and cholestatic liver disease] are quite frequent in daily medical activity. The proportion of patients affected by chronic liver diseases in different geographical areas can differ, in connection with the incidence of $\mathrm{B}$ and $\mathrm{C}$ hepatitis virus infections, with alcohol consumption, with the prevalence of obesity or

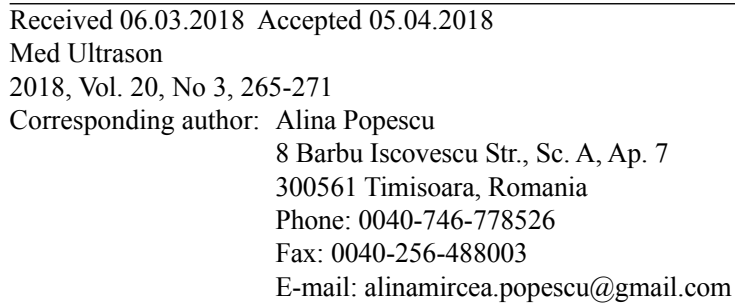

metabolic syndrome. Considering all these etiologic factors, $10 \%$ or more of the population is affected by chronic liver diseases [1]; therefore, how can we evaluate and follow-up these subjects? Liver biopsy is an option for the first evaluation, but further biopsies are very rarely performed (patients usually refusing a second biopsy), so what other modalities for liver assessment and prognosis can be used?

Non-invasive methods to assess the severity of chronic liver diseases have been available for more than 10 years in clinical practice and have become more and more popular, especially in Europe. Biological tests (easy to perform and not very expensive) and elastographic methods are used in daily practice.

Elastographic methods for liver fibrosis assessment can be divided into ultrasound based elastography and magnetic resonance elastography (MRE) [2]. MRE is more developed in USA and US based elastography in Europe and Asia. 
In the last few years, several ultrasound based elastographic methods (both strain and shear waves elastography - SWE - techniques) have become available in clinical use [3-6]. While contradictory results were obtained when strain elastography was used for liver fibrosis assessment [7-9], published papers and meta-analysis demonstrated that SWE techniques can be useful in clinical practice for fibrosis evaluation [10-12]. Currently, the number of non-invasive elastographic techniques have increased, each method having some limitations. The first elastographic technique used for liver fibrosis assessment was Transient Elastography (TE), followed by point SWE and, more recently, real time SWE (2D SWE). Even if all techniques are ultrasound based they are different and the interpretation of the results can be difficult in clinical practice.

The aim of our study was to evaluate the correlation of four shear waves elastographic methods in a cohort of patients with chronic liver diseases of mixed etiologies, and secondly, to compare the performances of three elastographic techniques used for liver fibrosis evaluation: point SWE - VTQ and ElastPQ, respectively and 2D SWE- SuperSonic Shear Imaging (SSI), considering Transient Elastography (TE) as the reference method.

\section{Material and methods}

\section{Patients}

A prospective study was conducted on 127 consecutive patients with chronic liver diseases of mixed etiologies. All subjects included agreed to undergo elastographic measurements and the study was approved by the Ethics Committee and was performed in accordance with the last revised version of the Helsinki Declaration.

All patients included were previously diagnosed with HBV, HCV, NASH or other non-viral hepatopathies. None of the included patients were currently under antiviral treatment. We excluded patients with ascites (because TE is not feasible in these patients), those with ultrasound signs of biliary obstruction and liver congestion secondary to heart failure, patients with elevated lever enzymes more than 3 times normal value and patients in whom focal liver lesions were found during abdominal ultrasound examination. We also excluded those patients in whom TE was not feasible with the M probe.

Liver stiffness (LS) measurements were performed in all subjects, after informed consent, in one session, during the same day, by means of 4 elastographic methods: Transient Elastography (TE) (FibroScan, EchoSens), Virtual Touch Quantification (VTQ) (Siemens Acuson S2000 ${ }^{\mathrm{TM}}$ ), ElastPQ (Philips, Affinity), and 2D-SWE.SSI
(Aixplorer, SuperSonic Imagine S.A). The enrolment period was of 4 months.

\section{Elastographic measurements}

All the examinations were performed in fasting conditions, with the patient in supine position, elastographic measurements being performed in the right liver lobe, through an intercostal space. For the elastographic methods with visual real time evaluation of the liver, the examination box was placed more than $1 \mathrm{~cm}$ below the capsule of the liver, to avoid falsely elevated stiffness measurements due to the closeness to the capsule. Minimal scanning pressure was applied by the operator while the patient was asked to stop breathing for a moment in order to minimize breathing motion. All elastographic measurements were performed by different experienced operators, with at least 6 months experience with the ultrasound system, who performed elastographic measurements blinded to all clinical and elastographic data obtained from the other elastographic techniques. All elastographic measurements were performed in the intercostal spaces choose by each operator as the best place for assessment, with the best acoustic window for the liver.

In all the patients the following parameters were documented: age, gender, body mass index (BMI), presence of chronic hepatopathies, presence of liver cirrhosis.

LS was measured by means of TE using a FibroScan ${ }^{\circledR}$ device (EchoSens, Paris, France), which incorporates an ultrasound transducer probe mounted on the axis of a vibrator. In each patient 10 valid TE measurements were performed and the median value was calculated. Reliable measurements were defined as: median value of 10 valid LS measurements with a success rate $(\mathrm{SR}=$ ratio of the number of successful acquisitions over the total number of acquisitions) $\geq 60 \%$ and an interquartile range (IQR=the difference between the 75 th and 25 th percentile, actually the range of the middle $50 \%$ of the data) $<30 \%$. The results were expressed in $\mathrm{kPa}$. Failure of TE measurements was defined as no valid measurement obtained after at least 10 shots, and a measurement was considered as unreliable in the following situations: fewer than 10 valid shots; $\mathrm{SR}<60 \%$ and $/$ or IQR $\geq 30 \%$ [13].

TE measurements were performed using the $M$ probe, by an experienced operator (more than 500 examinations), who was blinded to all clinical and elastographic data.

Since TE is a validated method for liver fibrosis evaluation, it was considered as the reference method, to which the performances of VTQ, ElastPQ, and 2DSWE.SSI elastography were compared. To discriminate between various stages of fibrosis we used the published 
LS cut-offs by TE proposed in the Tsochatzis et al (2011) meta-analysis [10] (Table I).

VTQ was performed with a Siemens Acuson S2000 Virtual TouchTM ultrasound system (Siemens AG, Erlangen, Germany) with a 4CI transducer. Ten valid VTQ measurements were performed in each patient, and the median value was calculated and expressed in meters/ seconds $(\mathrm{m} / \mathrm{s})$. One of the main advantages of this procedure is that the operator can select the region of interest in which liver stiffness is measured while performing real-time ultrasound, by placing the "measuring box" $(10 \times 5 \mathrm{~mm})$ in the desired place.

VTQ measurements was performed by an operator with 2 years experience in conventional ultrasound examination and more than 200 VTQ measurements performed, who was blinded to all clinical and elastographic data.

Initially, the VTQ technique manufacturer did not recommend the use of quality parameters IQR and SR, but a study published by our group [14] showed that the correlation of LS assessed by VTQ with fibrosis was significantly better in patients in whom VTQ measurements had quality technique parameters as compared with those with high IQR and/or low SR. Considering this observation, we used for VTQ the same criteria for defining failure, unreliable and valid measurements as for TE: reliable measurement - the median value of 10 valid LS measurements with a success rate $(\mathrm{SR}=$ ratio of the number of successful acquisitions over the total number of acquisitions) $\geq 60 \%$ and an interquartile range ( $\mathrm{IQR}=$ the difference between the 75th and 25th percentile, actually the range of the middle $50 \%$ of the data) $<30 \%$; unreliable measurement - fewer than 10 valid shots; SR $<60 \%$ and/or IQR $\geq 30 \%$.

To discriminate between various stages of fibrosis by VTQ we used LS cut-offs proposed by the Nierhoff meta-analysis [11] (Table I).

2D-SWE was performed with an Aixplorer ${ }^{\mathrm{TM}}$ ultrasound system (SuperSonic Imagine S.A., Aix-enProvence, France) (2D-SWE.SSI), using a SC6-1 convex probe.

In each patient 3 valid 2D-SWE.SSI measurements were performed, at least $2 \mathrm{~cm}$ under the liver capsule, in an area of parenchyma free of large vessels, in a homogenous region of the color coded map and using a $1 \mathrm{~cm}$ diameter circular region of interest. A median value of 3 valid 2D-SWE.SSI measurements was calculated, expressed in $\mathrm{kPa}$ [15]. No measurement quality criteria are recommended by the producer so none were used.

The 2D-SWE.SSI measurements were performed by an operator with more than 100 measurements performed with this machine, who was blinded to all clinical and elastographic data.
Table I. Cut-off values used for all four elastographic methods (TE, VTQ, ElastPQ and 2D-SWE.SSI)

\begin{tabular}{lll}
\hline & $\mathbf{F} \geq \mathbf{2}$ & $\mathbf{F}=\mathbf{4}$ \\
\hline TE [10] & $7 \mathrm{kPa}$ & $12 \mathrm{kPa}$ \\
VTQ [11] & $1.35 \mathrm{~m} / \mathrm{s}$ & $1.87 \mathrm{~m} / \mathrm{s}$ \\
2D-SWE.SSI [16] & $7.1 \mathrm{kPa}$ & $13 \mathrm{kPa}$ \\
ElastPQ [19] & $7.2 \mathrm{kPa}$ & $8.9 \mathrm{kPa}$ \\
\hline
\end{tabular}

$\mathrm{F}$ - fibrosis stage

For fibrosis estimation by means of 2D-SWE.SSI, we used the cut-offs proposed in the largest published study by Herrmann et al. (2015) which compared 2D-SWE.SSI measurements with liver biopsy (LB) [16] (Table I).

ElastPQ was performed with an Affiniti 70 ultrasound system (Philips, the Netherlands), using C5-1 PureWave Convex probe. The measurements were performed in a homogenous area, free of large vessels, at least $1 \mathrm{~cm}$, below the liver capsule. Ten valid measurements were performed and the median value was calculated and expressed in $\mathrm{kPa}$. Reliable measurements were considered the median value of 10 valid LS measurements with an interquartile range/median $<30 \%$ (IQR/Median $<30 \%$ ) recommended by The Society of Radiologists in Ultrasound [17] and proven by the Ferraioli at al study [18]. For fibrosis estimation we used the cutoffs proposed by Mare et al [19] (Table I).

\section{Statistical analysis}

The statistical analysis was performed using SPSS software, Version 20.0 (IBM SPSS Statistics), MedCalc Version 17.7.2 and Microsoft Office Excel 2007 (Microsoft for Windows).

Descriptive statistics was used for clinical, athropometric and demographic data of the patients. Numerical variables with normal distribution are presented as means \pm standard deviation, while variables with non-normal distribution are presented as median values and range. The Kolmogrov-Smirnov test was used for testing the distribution of numerical variables. Qualitative variables were presented as numbers and percentages. Parametric tests (t-test) were used for the assessment of differences between numerical variables with normal distribution; and nonparametric tests (Mann-Whitney or KruskalWallis tests) for variables with non-normal distribution. Chi-square $\left(\mathrm{X}^{2}\right)$ test (with Yates' correction for continuity) was used for comparing proportions expressed as percentages (" $n$ " designates the total number of patients included in a particular subgroup). $95 \%$ confidence intervals were calculated for each predictive test and a $p$-value $<0.05$ was considered as significant for each statistical test. The Spearman Test was used for correlations. For comparing the other techniques with TE we used the Pearson coefficient of precision and accuracy included in 
Lin's Concordance Correlation Coefficient (CCC). Lin's concordance correlation coefficient is a measure of how well a set of bivariate data compares to a "gold standard" measurement or test [20].

\section{Results}

Patient's characteristics are presented in Table II.

Valid LS measurements by all four shear waves ultrasound elastographic methods were obtained in 116/127 (92.1\%) subjects with VTQ, in 108/127 (95\%) subjects with 2D-SWE.SSI, in 111/127 (87.4\%) with TE and in $109 / 127(85.8 \%)$ with ElastPQ, so the final analysis included $82 / 127$ subjects $(64.5 \%)$. The patient's characteristics of this subgroup are presented in Table III.

Table II. Baseline characteristics of patients $(\mathrm{n}=127)$

\begin{tabular}{ll}
\hline Parameter & \\
\hline Age (years) & $51.3 \pm 14.1$ \\
Gender: & \\
$\quad$ - male & $\mathrm{n}=66(51.9 \%)$ \\
$\quad$ - female & $\mathrm{n}=61(48.1 \%)$ \\
Mean BMI (kg/m2) & $26.6 \pm 4.9$ \\
Diagnosis: & \\
$\quad$ - chronic hepatitis B & $\mathrm{n}=44(34.6 \%)$ \\
$\quad$ - chronic hepatitis C & $\mathrm{n}=49(38.5 \%)$ \\
$\quad$ - chronic non-viral hepatitis & $\mathrm{n}=23(18.1 \%)$ \\
$\quad$ NASH/ASH & $\mathrm{n}=11(8.8 \%)$ \\
Fibrosis stage (Fibroscan) & \\
$\quad$ F $<2$ & $\mathrm{n}=52(40.9 \%)$ \\
F2/F3 & $\mathrm{n}=33(25.9 \%)$ \\
F=4 & $\mathrm{n}=42(33.2 \%)$ \\
\hline
\end{tabular}

$\mathrm{n}$ - number of patients; BMI - body mass index; NASH-nonalcoholic steatohepatitis; ASH alcoholic steatohepatitis

Table III. Baseline characteristics of patients with valid liver stiffness measurements by all 4 shear waves ultrasound elastographic methods $(\mathrm{n}=82)$.

\begin{tabular}{ll}
\hline Parameter & \\
\hline $\begin{array}{l}\text { Age (years)* } \\
\text { Gender: }\end{array}$ & $49.5 \pm 15.2$ \\
$\quad$ - male & $\mathrm{n}=38(46.3 \%)$ \\
$\quad$ - female & $\mathrm{n}=44(53.65 \%)$ \\
BMI (kg/m2)* & $24.8 \pm 3.5$ \\
Diagnosis: & \\
$\quad$ - chronic hepatitis B & $\mathrm{n}=38(46.3 \%)$ \\
- chronic hepatitis C & $\mathrm{n}=28(34.1 \%)$ \\
$\quad$ - chronic non-viral hepatitis & $\mathrm{n}=9(10.9 \%)$ \\
- NASH/ASH & $\mathrm{n}=7(8.7 \%)$ \\
Fibrosis stage (Fibroscan) & $\mathrm{n}=46(56 \%)$ \\
$\quad \mathrm{F}<2$ & $\mathrm{n}=17(20.7 \%)$ \\
$\quad$ F2/F3 & $\mathrm{n}=19(23.3 \%)$ \\
$\quad$ F=4 & \\
*Mean \pm Standard deviation; $\mathrm{n}-$ number of patients; BMI - body \\
mass index; NASH - non alcoholic steatohepatitis; ASH - alco- \\
holic steatohepatitis
\end{tabular}

Higher BMI and older age were significantly associated with the impossibility of obtaining reliable LS measurements by TE. In 2D-SWE.SSI and VTQ elastography measurements, the presence of chronic hepatopathies, cirrhosis, older age, higher BMI were also associated with failed and unreliable measurements. The comparison between various elastography methods and TE showed different correlation in terms of both precision and accuracy (Table IV). A good and significant correlation was found between all 4 elastographic methods (fig 1).

The performance of VTQ, ElastPQ, and 2D-SWE.SSI for diagnosing significant fibrosis (F2/F3) and cirrhosis (F4) was evaluated using TE as a reference method in the subgroup of 82 subjects (a group formed by patients with valid liver stiffness measurements by all 4 shear waves

Table IV. Concordance analysis between other elastographic techniques and Transient Elastography

\begin{tabular}{llll}
\hline $\begin{array}{l}\text { Elastographic } \\
\text { method }\end{array}$ & $\begin{array}{l}\text { Precision } \\
\text { coefficient }\end{array}$ & $\begin{array}{l}\text { Accuracy } \\
\text { coefficient }\end{array}$ & CCC \\
\hline ElastPQ & 0.74 & 0.86 & 0.64 \\
VTQ & 0.80 & 0.89 & 0.72 \\
2D-SWE.SSI & 0.86 & 0.95 & 0.82 \\
\hline
\end{tabular}

CCC - Concordance correlation coefficient; VTQ - Virtual Touch Quantification; 2D-SWE - real time Shear Wave Elastography; 2DSWE.SSI - 2D-SWE from Aixplorer, SuperSonic Imagine S.A
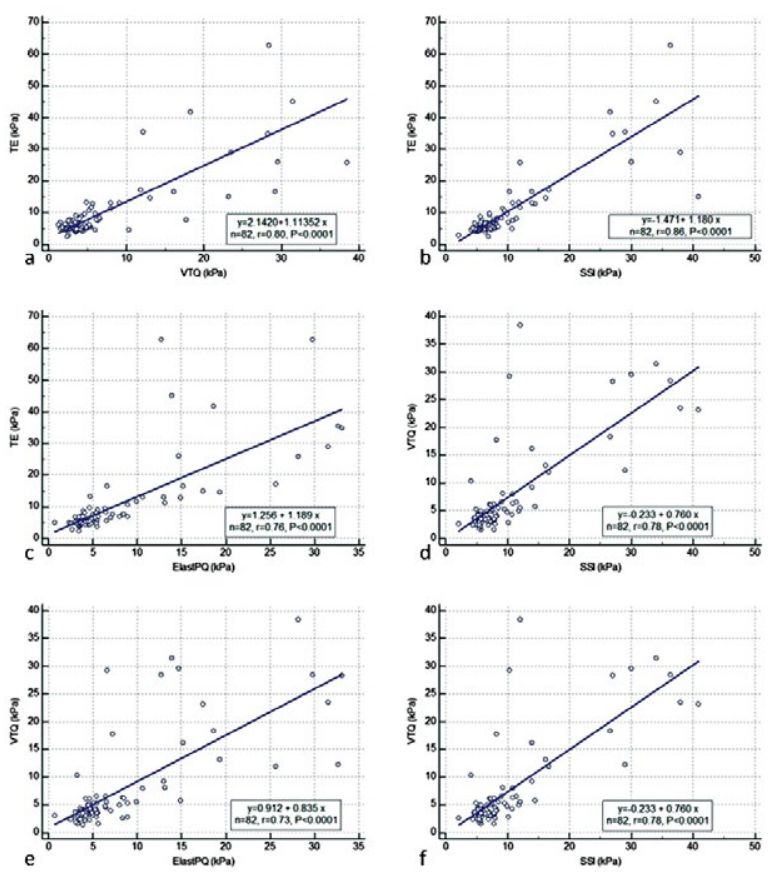

Fig 1. a) Comparison of TE versus VTQ; b) versus 2D-SWE. SSI; and c) versus ElastPQ; d) Comparison of VTQ versus 2DSWE.SSI; and e) versus ElastPQ; f) Comparison of 2D-SWE.SSI versus ElastPQ. Reports of the regression equation $(y=a+b x)$, sample size (n), Pearson's coefficient (r) and $p$ value (p). 
Table V. The performance of VTQ, 2D-SWE.SSI and ElastPQ for diagnosing liver fibrosis, using TE as ,gold-standard” for liver fibrosis assessment.

\begin{tabular}{lllllll}
\hline Fibrosis & Method & Sensibility (\%) & Specificity (\%) & PPV (\%) & NPV (\%) & Accuracy (\%) \\
\hline \multirow{2}{*}{ F $<2$} & VTQ & 79.5 & 90.9 & 92.8 & 70 & 82 \\
& 2D-SWE.SSI & 97.5 & 70.7 & 76.9 & 96.6 & 80 \\
& ElastPQ & 84.5 & 87.1 & 89.2 & 80.7 & 86 \\
F2/F3 & VTQ & 69.4 & 95.6 & 92.5 & 95.6 & 84.1 \\
& 2D-SWE.SSI & 96.8 & 78 & 73.8 & 97.5 & 85.3 \\
& ElastPQ & 77 & 90.1 & 87 & 82 & 84 \\
F4 & VTQ & 80.1 & 98.3 & 98.3 & 93.8 & 93.9 \\
& 2D-SWE.SSI & 78.9 & 97.7 & 88.2 & 95.5 & 94 \\
\hline
\end{tabular}

F - fibrosis stage; VTQ - Virtual Touch Quantification; 2D-SWE - real time Shear Wave Elastography; 2D-SWE.SSI - 2D-SWE from Aixplorer, SuperSonic Imagine S.A

ultrasound elastographic methods). Based on TE cut-off values, we divided our cohort into 3 groups: $\mathrm{F}<2$ : 46/82 (56\%); F2/F3: 17/82 (20.7\%); F=4: 19/82 (23.3\%).

The diagnostic accuracy of VTQ, 2D-SWE.SSI, and ElastPQ for the diagnosis of absence or mild fibrosis $(\mathrm{F}<2)(\mathrm{TE}<7 \mathrm{kPa})$, significant and severe fibrosis $(\mathrm{F} 2 /$ $\mathrm{F} 3),(7-12 \mathrm{kPa})$, and cirrhosis $(\mathrm{TE}>12 \mathrm{kPa})$ was similar for all elastographic techniques (table $\mathrm{V}$ ).

\section{Discussions}

Currently, many ultrasound systems able to perform liver elastography are available on the market. It can be difficult for practitioners to decide which is the best system with regard to the feasibility and accuracy of the method in evaluating the liver fibrosis. Feasibility is the first factor to consider when evaluating a method. The factors that usually impair the feasibility of elastographic techniques are obesity and advanced disease [21].

In our study we used TE as a reference method, since multiple studies had demonstrated its value for liver fibrosis assessment. TE is the only elastographic technique included in international guidelines for diagnosis and treatment of chronic hepatitis [22,23].

Previous studies evaluating the feasibility of TE in large cohorts of patients $[13,24]$ showed that TE has a high failure rate (especially when using only the $M$ probe), but using both $\mathrm{M}$ and XL probes, the feasibility can be improved to more than $90 \%$ [25,26]. For VTQ and ElastPQ, previous studies showed good feasibility $[14,27,28]$, while for 2D-SWE.SSI the results are contradictory $[29,30]$. In our comparative study, higher BMI and older age were significantly associated with the impossibility of obtaining reliable LS measurements by all four SWE methods. But when interpreting these results we must consider the fact that no techniqual quality criteria were used for 2D-SWE.SSI, since none were recommended by the producers. The consensus conference of the Society of Radiologists in Ultrasound (2015) [17] recommended the use of qualitative criteria for every elastographic technique.

Only a few papers evaluated the performance of several elastographic methods for staging fibrosis using liver biopsy as a reference method. Maybe the most important is the study published by Cassinotto et al [31]. In this study a cohort of 349 consecutive patients with chronic liver diseases were evaluated by liver biopsy, TE ( $M$ and XL probes), VTQ, and 2D-SWE.SSI. The correlation between the results of these elastographic techniques and LB were $\mathrm{r}=0.70, \mathrm{p}<00001$ for TE; $\mathrm{r}=0.64, \mathrm{p}<00001$ for VTQ; and $\mathrm{r}=0.79, \mathrm{p}<00001$ for SSI respectively. The AUROCs for TE, VTQ and 2D-SWE.SSI for the diagnosis of significant fibrosis $(\geq F 2)$ were $0.84,0.81$ and 0.88 , respectively, while for diagnosing cirrhosis they were $0.90,0.90$ and 0.93 , respectively.

The same group compared TE, VTQ, and 2D-SWE. SSI in a group of 291 patients with NAFLD (non-alcoholic fatty liver disease) [32], also considering LB as the gold standard. AUROCs for TE, VTQ and 2D-SWE.SSI were $0.82,0.77$ and 0.86 for $\geq \mathrm{F} 2$ and $0.87,0.84$ and 0.88 respectively for liver cirrhosis. Both Cassinotto's et al studies showed good results in predicting the severity of fibrosis for all three methods, with slight differences regarding accuracy, which improved with the severity of fibrosis.

A recent study compared three elastographic methods: TE, 2D-SWE, and ARFI-VTQ in patients with chronic hepatopathies. The reference methods were liver biopsy for the assessment of liver fibrosis and magnetic resonance imaging/computed tomography for the diagnosis of liver cirrhosis. No significant differences in diagnostic accuracy were found between the three elastographic techniques for the diagnosis of significant fibrosis, advanced fibrosis and also for liver cirrhosis [33].

In our cohort of subjects, the accuracy of VTQ, ElastPQ, and 2D-SWE.SSI (using TE as reference) showed 
comparable results, with an accuracy around $83 \%$ for significant fibrosis $(\geq \mathrm{F} 2)$ and around $93 \%$ for liver cirrhosis. For daily practice, these results are quite good; all these three methods (ElastPQ, VTQ and 2D-SWE.SSI) were able to assess the severity of liver disease. The accuracy increased with the severity of fibrosis, as demonstrated in many previous studies on all elastographic methods. For the assessment of significant fibrosis, the specificity and the negative predictive values are high, which are useful in daily practice. For liver cirrhosis, these discriminators are very high (more than $95 \%$ ) making the diagnosis very precise.

On the other hand, we found a good correlation between all 4 elastographic methods, quite similar with the results presented by other authors [34], that showed moderate to good correlation between different share wave elastographic methods and TE.

Limitations of our study are the facts that we could not use the Bland-Altman plot analysis, because our sample size was less than 100. Another limitation is that liver biopsy was not the reference method, and also that the evaluation was made only in patients without ascites since TE cannot be performed in this category of subjects. As a result, these limitations could introduce a bias, improving the performance of TE compared to the other methods. Another limitation is the fact that quality techniqual parameters were used only for TE, VTQ, ElastPQ and not for 2D-SWE.SSI, that could lead to a better feasibility for the latter. But no quality parameters were recommended by the producers. On the other hand the assessment methodology of this study did not specify that all measurements should be performed in the same intercostal space, but the study followed more the clinical practice where there is no recommendation regarding this issue. Our purpose was to compare the results of three ultrasound based elastographic methods (all integrated into ultrasound machines) with a validated elastographic method, used for a long time in many centers and to show the similarity of liver stiffness measurements performed with these devices. The decrease in the number of LBs performed as a result of the development of non-invasive techniques will lead to many future studies using other reference methods for fibrosis evaluation, rather than liver biopsy.

Considering the results of our study one question arises: should we use only TE in daily practice (since it was the first validated method), or could we use any of the other elastographic techniques for liver fibrosis evaluation? Probably the answer is that any of these SWE methods can be used in daily practice [35], with good accuracy. As opposed to TE, SWE methods can be performed in patients with ascites and are integrated into ultrasound machines which can perform a standard ul- trasound examination, Doppler examination, or Contrast Enhanced Ultrasound examinations, besides elastographic measurements.

In conclusion considering TE as the reference method for liver fibrosis evaluation, VTQ, ElastPQ and 2DSWE.SSI obtained similar accuracies for diagnosing at least significant fibrosis $(\mathrm{F} \geq 2)$ and liver cirrhosis.

\section{Conflict of interest: none}

\section{References}

1. Blachier M, Leleu H, Peck-Radosavljevic M, Valla D, Roudot-Thoraval F. The burden of liver disease in Europe: A review of available epidemiological data. J Hepatol 2013;58:593-608.

2. Wang QB, Zhu H, Liu HL, Zhang B. Performance of magnetic resonance elastography and diffusion-weighted imaging for the staging of hepatic fibrosis: A meta-analysis. Hepatology 2012;56:239-247.

3. Bamber J, Cosgrove D, Dietrich CF, et al. EFSUMB Guidelines and Recommendations on the Clinical Use of Ultrasound Elastography. Part 1: Basic Principles and Technology. Ultraschall Med 2013;34:169-184.

4. Cosgrove D, Piscaglia F, Bamber J, et al. EFSUMB Guidelines and Recommendations on the Clinical Use of Ultrasound Elastography.Part 2: Clinical Applications. Ultraschall Med 2013;34:238-253.

5. Shiina T, Nightingale KR, Palmeri ML, et al. WFUMB Guidelines and Recommendations for Clinical Use of U1trasound Elastography: Part 1: Basic Principles and Terminology. Ultrasound Med Biol 2015;41:1126-1147.

6. Ferraioli G, Filice C, Castera L, et al. WFUMB Guidelines and Recommendations for Clinical Use of Ultrasound Elastography: Part 3: Liver. Ultrasound Med Biol 2015;41:1161-1179.

7. Friedrich-Rust M, Ong MF, Herrmann E, et al. Real-Time Elastography for Noninvasive Assessment of Liver Fibrosis in Chronic Viral Hepatitis. AJR Am J Roentgenol 2007; 188:758-764.

8. Ferraioli G, Tinelli C, Malfitano A, et al. Performance of Real-Time Strain Elastography, Transient Elastography, and Aspartate-to-Platelet Ratio Index in the Assessment of Fibrosis in Chronic Hepatitis C. AJR Am J Roentgenol 2012;199:19-25.

9. Yada N, Kudo M, Morikawa H, Fujimoto K, Kato M, Kawada N. Assessment of liver fibrosis with real-time tissue elastography in chronic viral hepatitis. Oncology 2013;84:13-20.

10. Tsochatzis EA, Gurusamy KS, Ntaoula S, et al. Elastography for the diagnosis of severity of fibrosis in chronic liver disease: A meta-analysis of diagnostic accuracy. J Hepatol 2011;54:650-659.

11. Nierhoff J, Chávez Ortiz AA, Herrmann E, Zeuzem S, Friedrich-Rust M. The efficiency of acoustic radiation force 
impulse imaging for the staging of liver fibrosis: a metaanalysis. Eur Radiol 2013;23:3040-3053.

12. Bota S, Herkner H, Sporea I, et al. Meta-analysis: ARFI elastography versus transient elastography for the evaluation of liver fibrosis. Liver Int 2013;33:1138-1147.

13. Castéra L, Foucher J, Bernard PH, et al. Pitfalls of liver stiffness measurement: A 5-year prospective study of 13,369 examinations. Hepatology 2010;51:828-835.

14. Bota S, Sporea I, Sirli R, Popescu A, Dănilă M, Sendroiu $M$. Factors that influence the correlation of acoustic radiation force impulse (ARFI), elastography with liver fibrosis. Med Ultrason 2011;13:135-140.

15. Sporea I, Grădinaru-Taşcău O, Bota S, et al. How many measurements are needed for liver stiffness assessment by $2 \mathrm{D}$-Shear Wave Elastography (2D-SWE) and which value should be used: the mean or median? Med Ultrason 2013;15:268-272.

16. Herrmann E, de Lédinghen V, Cassinotto C, et al. Assessment of biopsy-proven liver fibrosis by 2D-shear wave elastography: An individual patient data based meta-analysis. Hepatology 2018;67:260-272.

17. Barr RG, Ferraioli G, Palmeri ML, et al. Elastography Assessment of Liver Fibrosis: Society of Radiologists in Ultrasound Consensus Conference Statement. Radiology 2015;276:845-861.

18. Ferraioli G, Maiocchi L, Lissandrin R, et al; Liver Fibrosis Study Group. Accuracy of the ElastPQ Technique for the Assessment of Liver Fibrosis in Patients with Chronic Hepatitis C: a "Real Life" Single Center Study. J Gastrointestin Liver Dis 2016;25:331-335.

19. Mare R, Sporea I, Lupusoru R, et al. The value of ElastPQ for the evaluation of liver stiffness in patients with $\mathrm{B}$ and $\mathrm{C}$ chronic hepatopathies. Ultrasonics 2017;77:144-151.

20. Lin LI. A concordance correlation coefficient to evaluate reproducibility. Biometrics 1989;45:255-268.

21. Pang JX, Pradhan F, Zimmer S, et al. The feasibility and reliability of transient elastography using Fibroscan ${ }^{\circledR}$ : a practice audit of 2335 examinations. Can J Gastroenterol Hepatol 2014;28:143-149.

22. European Association for Study of Liver; Asociacion Latinoamericana para el Estudio del Higado. EASL-ALEH Clinical Practice Guidelines: Non-invasive tests for evaluation of liver disease severity and prognosis. J Hepatol 2015;63:237-264.

23. European Association for Study of Liver. EASL Recommendations on Treatment of Hepatitis C 2015. J Hepatol 2015;63:199-236.
24. Şirli R, Sporea I, Bota S, Jurchiş A. Factors influencing reliability of liver stiffness measurements using transient elastography (M-probe) - monocentric experience. Eur J Radiol 2013;82:e313-e316.

25. Şirli R, Sporea I, Deleanu A, et al. Comparison between the $\mathrm{M}$ and XL probes for liver fibrosis assessment by transient elastography. Med Ultrason 2014;16:119-122.

26. Sporea I, Sirli R, Mare R, Popescu A, Ivascu SC. Feasibility of Transient Elastography with M and XL probes in real life. Med Ultrason 2016;18:7-10.

27. Sporea I, Bota S, Jurchis A, et al. Acoustic radiation force impulse and supersonic shear imaging versus transient elastography for liver fibrosis assessment. Ultrasound Med Biol 2013;39:1933-1941.

28. Ferraioli G, Tinelli C, Lissandrin R, Zicchetti M, Dal Bello B, Filice C. Performance of ElastPQ ${ }^{\circledR}$ shear wave elastography technique for assessing fibrosis in chronic viral hepatitis. J Hepatol 2013;58:S7.

29. Sporea I, Bota S, Gradinaru-Taşcău O, Sirli R, Popescu A, Jurchis A. Which are the cut-off values of 2D-Shear Wave Elastography (2D-SWE) liver stiffness measurements predicting different stages of liver fibrosis, considering Transient Elastography (TE) as the reference method? Eur J Radiol 2014;83:e118-e122.

30. Bavu E, Gennisson JL, Couade M, et al. Noninvasive in vivo liver fibrosis evaluation using supersonic shear imaging: a clinical study on 113 hepatitis $C$ virus patients. Ultrasound Med Biol 2011;37:1361-1373.

31. Cassinotto C, Lapuyade B, Mouries A, et al. Non-invasive assessment of liver fibrosis with impulse elastography: Comparison of Supersonic Shear Imaging with ARFI and FibroScan ${ }^{\circledR}$. J Hepatol 2014;61:550-557.

32. Cassinotto C, Boursier J, de Lédinghen V, et al. Liver stiffness in nonalcoholic fatty liver disease: A comparison of supersonic shear imaging, FibroScan, and ARFI with liver biopsy. Hepatology 2016;63:1817-1827.

33. Gerber L, Kasper D, Fitting D, et al. Assessment of Liver Fibrosis with 2-D Shear Wave Elastography in Comparison to Transient Elastography and Acoustic Radiation Force Impulse Imaging in Patients with Chronic Liver Disease. Ultrasound Med Biol 2015;41:2350-2359.

34. Piscaglia F, Salvatore V, Mulazzani L, et al. Differences in liver stiffness values obtained with new ultrasound elastography machines and Fibroscan: A comparative study. Dig Liver Dis 2017;49:802-808.

35. Sporea I. One or more elastographic methods for liver fibrosis assessment? Med Ultrason 2015;17:137-138. 